NASA Technical Memorandum 101409

AIAA-89-0172

\title{
Axisymmetric Confined Turbulent Jet Directed Towards the Liquid Surface From Below
}

Mohammad M. Hasan

Lewis Research Center

Cleveland, Ohio

and

Chin-Shun Lin

Analex Corporation

Lewis Research Center

Cleveland, Ohio

Prepared for the

27th Aerospace Sciences Meeting

sponsored by the American Institute of Aeronautics and Astronautics

Reno, Nevada, January 9-12, 1989 


\title{
AXISYMMETRIC CONFINED TURBULENT JET DIRECTED TOWAROS THE LIQUID SURFACE FROM BELOW
}

\author{
Mohammad M. Hasan \\ National Aeronautics and Space Administration \\ Lewis Research Center \\ Cleveland, Ohio 44135 \\ and \\ Chin-Shun Lin \\ Analex Corporation \\ Lewis Research Center \\ Cleveland, Ohio 44135
}

\section{Abstract}

The paper presents a numerical solution of an axisymmetric turbulent jet discharging axially from below into a cylindrical tank and directed towards the liquid vapor interface. The liquid-vapor interface is assumed to be flat and shear free. The $k-\varepsilon$ turbulence model is used to calculate the eddy viscosity. The turbulence intensity distribution and the length scale associated with the $k-\varepsilon$ model are caiculated as functions of jet flow rates and system parameters. Numerical results are compared with appropriate experimental data. The problems associated with the free surface boundary conditions for the turbulent quantities are discussed.

\section{Nomenc lature}

$c_{1}, c_{2}, c_{\mu}$

constants in $k-\varepsilon$ turbulence model

D

d

G

g

k

L

$\mathrm{p}$

Q

R

$r$

$\operatorname{Re}_{j}$

U

$u$

v

v

$x$ $x_{5}$

y

Greek symbols:

$\varepsilon$

$\kappa$

$\Lambda$

(1)

$\mu$

$\mu t$

Heff effective viscosity

$\rho$

$\sigma_{k}$

$\sigma_{\varepsilon}$

\section{ORIGINAL PAGR IS OF POOR QUALTTY}

liquid viscosity

eddy viscosity

liquid density

Prandtl number in $k$ equation

$\sigma_{\varepsilon} \quad$ Prandtl number in $\varepsilon$ equation
Subscripts:

S

Background

The present work is motivated by the fluid mixing and the liquid-vapor interface condensation problems which may be encountered during the onorbit storage and transfer of subcritical cryogens and the pressure control of the storage tank. The liquid-vapor interface location and configuration are not well defined in micro-gravity environments. Therefore, direct venting of vapor to control the tank pressure either during long-term storage or during the filling process of an empty tank is not a viable option. The problems associated with direct venting in low-gravity conditions are adequately described by Labus et al. I Realization of these problems has led to the development of the concept of thermodynamic vent system ${ }^{-5}$ (TVS) for the pressure control of cryogenic storage tanks and the "no-vent" fill technique 5,6 for filling tanks with cryogens. The rate of vapor condensation on to a liquid surface and the fluid mixing process are important problems associated with these systems. 
Fluid Mixing

Experimental investigations ${ }^{7-9}$ of fluid mixing due to an axial dyed jet introduced into an isothermal fluid (ethanol), were performed in both normal and reduced gravity environments. Based on the findings of these tests, axial liquid jets are considered as the preferred technique for providing fluid mixing in low-gravity conditions. The desired objective of the use of axial liquid jet is to control the tank pressure by destroying thermal stratification of the stored liquid and by enhancing the rate of condensation of the vapor on the liquidvapor interface.

\section{Vapor Condensation on the Liquid Surface}

Experimental findings have established that the rate of the vapor condensation on to the liquid can be greatly enhanced by the introduction of turbulence beneath the liquid surface. Sonin et al.10 and Helmick et al. 11 measured the steady state rate of condensation of nearly saturated steam onto a turbulent liquid surface. The turbulence on the liquid side was produced by a single axial jet directed towards the liquid surface from below. The geometrical configuration shown in Fig. I is an adaptation of the test cell used by Sonin et al.10 For a fixed water level, condensation rates were experimentally determined as a function of liquid side turbulence intensity. The turbulent intensity was controlled by varying the jet flow rate. The turbulence intensity $v$ (rms value of fluctuating velocity), its spatial distribution and the turbulence macro scale, were measured in the absence of condensation. These measurements were performed at axial locations greater than 30 from the jet inlet. It was argued and subsequently supported by experimental measurement, that in the upper region $(x / D$ ) 3 ), the turbulent velocities are much larger than any mean circulatory velocities. It was also established that at some distance below the interface (away from the damped layer) the turbulence is approximately isotropic in the horizontal plane and is essentially unaffected by the proximity of the interface, and the length scale of turbulence is locked to the system diameter, $D$. The most significant finding of these investigations 10,11 is that for a shear-free and wave-free liquid interface, the condensation rate can be characterized in terms of the liquid-side turbulence characteristics $v$ and $\Lambda$. Empirical correlations to describe the condensation rate, the turbulence intensity near the surface, and the conditions under which they apply are fulliy discussed in Refs. 10 and 11 . The objective of the present work is to compute turbulence characteristics of the physical system of Refs. 10 and 11 by solving the appropriate governing equations numerically.

\section{Problem Formulation}

\section{The Physical Problem}

The geometric configuration and the coordinates system used to analyze the problem are shown in Fig. 1. A cylindrical tank of diameter $D$ is partially filled with liquid. A circular liquid jet of diameter d directed upward along the tank central axis is introduced at the bottom of the tank.
Liquid is withdrawn from the outer part of the bottom of the tank. The volume flow rate of the liquid withdrawn is the same as that of the injected liquid. Therefore, the liquid level above the jet remains constant. The outflow area is made much larger than the inflow jet area, so that the outflow velocity is negligibly small compared with the jet velocity. However, in numerical computation the outflow velocity term is retained, even though it is two orders of magnitude lower than the jet inlet velocity. The injected liquid and the tank liquid temperatures are assumed to be the same and uniform. Therefore, the effects of heat transfer and thermal stratification on the flow field and the evolution of turbulence are not considered in the present analysis.

The geometric parameters of the problem are the ratios of the tank diameter to jet diameter, $D / d$, and the liquid height above the jet to the tank diameter $x_{5} / D$. In the present analysis only the parameter $x_{s} / D$ is varied.

\section{Governing Equations}

The jet considered in the present analysis is an axisymmetric turbulent jet. The flow is steadystate and incompressible. The liquid-vapor interface is assumed to be flat and shear free. The $k-\varepsilon$ model of Jones and Launder 12 is chosen for $[a]-$ culating the eddy viscosity. The time-averaged continuity, momentum and $k-\varepsilon$ equations are:

Conservation of mass

$$
\frac{\partial}{\partial x}(\rho U)+\frac{1}{r \partial r}(r \rho V)=0
$$

Conservation of $x$-momentum

$\frac{\partial}{\partial x}\left(\rho U^{2}\right)+\frac{\partial}{r \partial r}(r \rho V)=\frac{\partial}{\partial x}\left(\mu_{e f f} \frac{\partial U}{\partial x}\right)+\frac{\partial}{r \partial r}\left(r \mu_{e f f} \frac{\partial U}{\partial r}\right)$ $+\left\{-\frac{\partial p}{\partial x}+\rho g+\frac{\partial}{\partial x}\left(\mu_{e f f} \frac{\partial U}{\partial x}-\frac{2}{3} \rho k\right)+\frac{\partial}{r \partial r}\left(r \mu_{e f f} \frac{\partial V}{\partial x}\right)\right\}$

Conservation of $r$-momentum

$$
\begin{aligned}
\frac{\partial}{\partial x}(\rho U V) & +\frac{\partial}{r \partial r}\left(r \rho V^{2}\right)=\frac{\partial}{\partial x}\left(\mu_{\text {eff }} \frac{\partial V}{\partial x}\right) \\
+ & \frac{\partial}{r \partial r}\left(r \mu_{e f f} \frac{\partial V}{\partial r}\right)+\left\{-\frac{\partial p}{\partial r}+\frac{\partial}{\partial x}\left(\mu_{\text {eff }} \frac{\partial U}{\partial r}\right)\right. \\
+ & \left.\frac{\partial}{r \partial r}\left(r \mu_{\text {eff }} \frac{\partial V}{\partial r}-\frac{2}{3} \rho r k\right)-2 \mu_{\text {eff }} \frac{V}{r^{2}}\right\}
\end{aligned}
$$

\section{$k-\varepsilon$ turbulence model}

$$
\begin{aligned}
\frac{\partial}{\partial x}(\rho U k) & +\frac{1}{r \partial r}(r \rho U k)=\frac{\partial}{\partial x}\left[\left(\mu+\frac{\mu_{t}}{\sigma_{k}}\right) \frac{\partial k}{\partial x}\right] \\
+ & \frac{\partial}{r \partial r}\left[r\left(\mu+\frac{\mu_{t}}{\sigma_{k}}\right) \frac{\partial k}{\partial r}\right]+\left\{\mu_{e f f} G-\rho \varepsilon\right\}
\end{aligned}
$$




\section{ORIGINAL PAGE IS \\ OF. POOR QUALITY}

$$
\begin{aligned}
& \frac{\partial}{\partial x}(\rho U \varepsilon)+\frac{1}{r \partial r}\left(r \rho U_{\varepsilon}\right)=\frac{\partial}{\partial x}\left[\left(\mu+\frac{\mu_{t}}{\sigma_{\varepsilon}}\right) \frac{\partial \varepsilon}{\partial x}\right] \\
& +\frac{\partial}{r \partial r}\left[r\left(\mu+\frac{\mu_{t}}{\sigma_{\varepsilon}}\right) \frac{\partial \varepsilon}{\partial r}\right]+\left\{\frac{\varepsilon}{k}\left(C_{1} G_{e f f}-C_{2} \rho \varepsilon\right)\right\}
\end{aligned}
$$

where the turbulence production term, $G$, is defined by

$$
G=2\left[\left(\frac{\partial U}{\partial x}\right)^{2}+\left(\frac{\partial V}{\partial r}\right)^{2}+\left(\frac{V}{r}\right)^{2}\right]+\left(\frac{\partial U}{\partial r}+\frac{\partial V}{\partial x}\right)^{2}
$$

The terms in the braces, on the right-hand side of each equation are the source terms. The turbulent viscosity is given by the expression:

$$
\mu_{t}=C_{\mu} \frac{\rho k^{2}}{\varepsilon} ; \quad \mu_{e f f}=\mu+\mu_{t}
$$

Numerical values of the empirical constants associated with the turbulence model are 12,13

$$
\begin{aligned}
& c_{1}=1.44, \quad c_{2}=1.92, \quad c_{\mu}=0.09, \\
& \sigma_{k}=1.0, \quad \kappa=0.42 \text { and } \quad \sigma_{\varepsilon}=\frac{\kappa^{2}}{\left(c_{2}-c_{1}\right) c_{\mu}^{1 / 2}}
\end{aligned}
$$

\section{Boundary Conditions}

The flow doma in between $0 \leq r \leq D / 2$, and $0 \leq x \leq x_{s}$ is considered for the computations. The flow problem considered in the present analysis has a line on symmetry along the tank central axis. The symmetric conditions at the center line are:

$$
\frac{\partial U}{\partial r}=\frac{\partial k}{\partial r}=\frac{\partial \varepsilon}{\partial r}=0, \quad \text { and } \quad V=0
$$

At the wall no-slip boundary conditions apply

$$
U=V=0 \text {. }
$$

Also, the wall functions 14 are used to calculate shear.stress and are included as a source term for velocity component parallel to the wall in the governing equations.15

The velocity and the turbulent kinetic energy are assumed uniform at the jet inlet.

$$
U=U_{j}, \quad V=0 \text { and } k=0.005 U_{j}^{2}
$$

The distribution of the dissipation rate is given by 6 :

$$
\varepsilon=c_{\mu}^{3 / 4} \frac{k^{3 / 2}}{1}
$$

Where the mixing length scale, 1 , is given by the expressions:

$$
\begin{aligned}
1=k y \text { for } y & \leq\left(\frac{d}{2}\right)\left(\frac{C_{\mu}}{\kappa}\right) \text { where } y=\frac{d}{2}-r \\
1 & =C_{\mu}\left(\frac{d}{2}\right) \text { for } y \geq\left(\frac{d}{2}\right)\left(\frac{C_{\mu}}{\kappa}\right) .
\end{aligned}
$$

At the bottom of the tank (i.e., $x=0$ ), where the liquid is withdrawn, the velocity is assumed to be uniform and equal to $Q / A$, where $Q$ is the jet inflow rate and $A$ is the tank outflow area.

The boundary conditions for the turbulence quantities, $k$ and $\varepsilon$ at the liquid vapor interface, pose special problems in the numerical computation. To our knowledge, the appropriate boundary conditions for $k$ and $\varepsilon$ at the interface have not been established either theoretically or empirically. In the present analysis it is assumed that at the free surface

$$
\frac{\partial k}{\partial x}=\frac{\partial \varepsilon}{\partial x}=0
$$

Also, the assumption of the flat and shear free interface implies:

$$
U=0, \quad \frac{\partial V}{\partial x}=0 \quad \text { at } \quad x=x_{s}
$$

\section{Numerical Solution}

\section{Solution Methodology}

The governing equations (Eqs. (1) to (5)) were solved by a finite difference numerical scheme. 15,16 The finite-difference equations were derived by integrating the differential equations over an elementary control volume surrounding a grid node appropriate for each dependent variable. A staggered grid system was used. The scalar properties $P, k$ and $\varepsilon$ were stored midway between the $U$ and $V$ velocity grid nodes. The bounded skew hybrid differencing 16 was incorporated for the convective terms and the integrated source terms were linearized. The pressure distribution was obtained by means of pressure implicit split operation (PISO) 17 predictor-corrector technique.

The calculations were performed with a nonuniform grid distribution with the concentration of grid nodes near the center line, the wall and the interface regions where the gradients of flow properties are expected to be large. The grid independent solutions were determined by trial runs. A 36 by 31 grid in the $x$ and $r$ direction, respectively, was used for $x_{s} / D=3.67$. Comparison of numerical results of this grid size with that obtained by using higher grid numbers shows that the solutions are essentially grid independent. For $x_{5} / D$ greater that 3.67 , higher grid numbers were used in the $x$-direction ( 56 by 31 , for $\left.x_{S} / D=7.2\right)$.

The finite difference equations were solved iteratively by the ADI method with under relaxation 15 until the solution converged. The maximum absolute residual sums for each dependent variable less than 0.005 was used as a criterion of convergence. Based on this criterion, the convergent 
solutions were obtained within 1500 iterations, with $0.8 \mathrm{sec} /$ iteration on CRAY-XMP CPU time for initial guessed solutions set of zero.

\section{Parameters}

The numerical solutions presented in this paper were performed using the input conditions representative to those of experiments of Refs. 10 and 11 . The thermophysical properties of liquid water at $20{ }^{\circ} \mathrm{C}$ were used in all computations. The tank diameter, $D$, and the jet diameter, $d$, were set equal to 15.3 and $0.64 \mathrm{~cm}$, respectively (same as those of Ref. 10). The jet Reynolds numbers were varied between 30000 to 110000 , depending upon the liquid height to the tank diameter ratios, $x_{s} / D$. Most of the calculations were performed for $x_{s} / D$ of 3.67 and 4.65 . For high jet Reynolds number, numerical solutions were also obtained for $x_{s} / D=7.2$, to investigate what effect, if any, the large liquid depth has on the solution.

\section{Results and Discussion}

The numerical solutions of the differential Eqs. (1) to (5), with the empirical coefficients of the turbuience model given by Eq. (8), subject to the boundary conditions (9) to (15), yielded distributions of mean flow velocities $U$ and $V$, the turbulent $k i n e t i c$ energy, $k$, and the dissipation of the turbulence kinetic energy, $\varepsilon$. As noted in the preceding section, the geometrical configuration and the input conditions used in the present analysis are similar to those of the experiments. 10,11 Therefore, the numerical results that offer direct comparison with the experiments are presented in this paper. A detailed analys is of the numerical results encompassing a wide range of parameters is still being performed and will be reported in the future.

The numerical results for the rms value of the turbulence velocity, $v$, (calculated from the expression $\left.k=(3 / 2) v^{2}\right)$ at a fixed axial location, $x / D=3.33$, and two radial locations $r / R=0$ and $r / R=0.62$, are presented in Fig. 2, where they are plotted as a function of the jet flow rate. The abscissa is chosen as Q/Dd, to facilitate comparison with the experiment. The experimental rms values 10 of either the vertical or horizontal fluctuating velocity are shown on the figure for comparison. The range of jet Reynolds numbers for the stated range of flow rates varies between 30000 to 80000 . The liquid height to the tank diameter ratio, $x_{s} / 0$, for the experiment was 3.67 . The numerical results presented in Fig. 2 correspond to this value of $x_{s} / 0$. The horizontal sampling region for the experimental data extended from the axis to the $2 / 3$ radius of the test cell. The experimental data show that for $x / D>3$, and at a istance of the damped layer thickness $(\simeq 0.30)$ below the interface, the turbulence is essentially isotropic in a horizontal plane. The numerical results obtained by using the $k-\varepsilon$ model are shown in Fig. 2. The solid lines drawn in Fig. 2 show the numerical results of $v$ at the axis and at a radial distance of $0.62 \mathrm{R}$. Almost all of the measured data fall between these two lines and show excellent comparison with the numerical results. Both the experimental as well as the numerical results show almost a linear relation between $v$ and Q/Dd.
Figure 3 shows the variation of the turbulence velocity along the central axis as a function of distance from the interface. The measured values of the vertical and horizontal fluctuating velocities as reported in Ref. 11 are included for comparison. Both the measured and calculated results correspond to jet Reynolds number of 51000 and $x_{S} / 0=3.67$. The experimental results suggest that a depth of about $0.13 \mathrm{D}$ below the interface the turbulence is approximately isotropic and is essentially unaffected by the interface. The numerical results are in excellent agreement with the experiment in this region. The vertical turbulent velocity closer to the surface damps out. Its kinetic energy is imparted to the horizontal components with a resultant increase in the horizontal turbulent velocities. Therefore, the turbulence closer to the surface is nonisotropic and the use of $k-\varepsilon$ model may not be appropriate. The comparison between the numerical results and experimental data worsens as the free surface is approached.

Sonin, et al.10 obtained an approximate analytical solution for the turbulence distribution by applying the high Reynolds number form of the $k-\varepsilon$ model of turbulence to the region $x / D>3$, where the mean circulatory velocity is small compared with fluctuating velocities. They assumed that the turbulent kinetic energy, $k$, can be approximated as a function of $x$ alone and the length scale $\mathrm{L}=\mathrm{k} / 2 / \varepsilon$ in the model is constant and locked to the tube diameter. The analytical solution of the $k-\varepsilon$ model for purely diffusive turbulence shows that the isotropic turbulent velocity and the length scale are given by the expressions:

$$
v=b\left(\frac{Q}{D d}\right) \exp \left(\frac{-1 \cdot 36 x}{\beta D}\right)
$$

and

$L=\frac{k^{3 / 2}}{\varepsilon}=\beta D$ where $b$ and $\beta$ are constants

By comparing this solution with the experimental results Sonin et al. 10 suggested the values of $B=1.1$ and $b=21.8$ for high Reynolds number flows.

The experimental datalo used to $f i x$ the value of $\beta$ are reproduced in Fig. 4 along with the numerical solution of the corresponding problem. The numerical results show that the slope of $\ln (v)$ versus $x / D$ is not constant, which implies that $B$ is not a constant in the range of $3.1<x / 0<4.2$ as suggested in Ref. 10. Analysis of numerical results shows that for $x / D$, up to approximately 3.6 , the generation term in $k-\varepsilon$ equations is of the same order of magnitude as the diffusion term. Therefore, the solution obtained for purely diffusive turbulence may not apply in this region. The length scale, $L$ is plotted in Fig. 5 as a function of the radial distance from the axis. Figure 5 shows that the length scale at a given axial location is essentially uniform except close to the tank wall. However, the length scale varies with the axial distance which is not in agreement with Sonin, et al. 10 conclusion that 
for high Reynolds number the length scale is constant and locked to the system diameter. Near the surface, the numerical solution for the jet Reynolds number of 50000 shows that $L / D$ varies between 0.42 and 0.6 .

The analytical solution obtained in Ref. 10 should indeed be valid if $x$ is sufficiently large (theoretically $x \rightarrow \infty$ ). In order to investigate the effects of the liquid depth and the boundary conditions (14) for $k$ and $\varepsilon$ imposed at the interface, numerical solutions for a fixed jet Reynolds number of 110000 were obtained for liquid depths of $x_{5} / D=4.65$ and 7.2. The variation of the turbulent kinetic energy, $k$, and the length scale, $L$, as a function of $x / D$ is shown in Figs. 6 and 7. As seen from these figures the solution remains practically unaffected for $x / D$ up to 3.6 , and the length scale $L / D$ attains almost a constant value 0.9 for large $x / D$. The region $3.6<x / 0<4.6$ probably represents transition to purely diffusive turbulence where the effect of mean circulatory is negligible. However, the numerical results show that turbulent kinetic energy in steady diffusive turbulence region increases or approximately remain constant with the axial distance, $x$, which is unrealistic. In our opinion, it is caused by the imposition of the boundary conditons of $a / \partial x$ of all turbulence quantities zero at the interface. Also the constants incorporated in the model may not be appropriate. In the damped layer, close to the surface where the vertical component of turbulent velocity damps out, the use of the Reynolds-Stress equations may be useful. However, before a particular model is chosen, we feel that it is more important to resolve first, the issues associated with the free surface boundary condtions for the turbulence quantities.

\section{Conclusions}

The $k-\varepsilon$ model of turbulence is used to obtain numerical solutions of an axisymmetric turbulent jet discharging axially into a cylindrical tank and directed towards the 1 iquid vapor interface. The numerical results presented in the paper were performed using input conditions representative to those of Refs. 10 and 11 . In the region where the turbulence is approximately isotropic, the calculated values of the turbulent velocity compare well with the experiment.

The calculated values of the length scale. $L=k^{3 / 2} / \varepsilon$ do not agree with the conclusion and analytical solution of Ref. 10 . If the interface is far away from the jet, approximately $x_{S} / D>4.5$, it does not affect the solution up to $x / 0 \simeq 3.6$. However, the boundary conditions for $k$ and $\varepsilon$ imposed at the interface affect the solution in the entire region of diffusive turbulence. The results obtained by using the boundary conditions (14) for large $x_{s} / D$ apprear to be unrealistic.

\section{References}

1. Labus, T.L., Aydelott, J.C., and Amling, G.E., "Zero-Gravity Venting of Three Refrigerants," NASA TN D-7480, 1974.

2. Bullard, B.R., "Liquid Propellant Thermal Conditioning System Test Program," LMSC-DI59262, Lockheed Missiles and Space Co., Sunnyvale, CA, July 1972, NASA CR-72971.
3. Robinson, J:W., Giellis, R.T, and Fester, D.A., "Long Term Cryogentc Storage Study," AFRPL-TR-83-082, 1984. (Avail. NTIS. AD-B089763).

4. Aydelott, J.C., Carney, M.J., and Hochste in, J.I, "NASA Lewis Research Center Low-Gravity Fluid Management Technology Program," AIAA/GNOS Paper 85-002, Nov. 1985. (NASA TM-87145).

5. Merino, F., Blatt, M.K., and Thies, N.C., "Filling of Orbital Fluid Management Systems," CASD-NAS-78-010, General Dynamics/Convair, San Diego, CA, July 1978, NASA CR-159404.

6. Chato, D.J., "Thermodynamic Modeling of the No-Vent Fill Methodology for Transferring Cryogens in Low Gravity," AIAA-Paper 88-3403, July 1988. (NASA TM-100932).

7. Aydelott, J.C., "Axial Jet Mixing of Ethanol in Spherical Containers During Weightlessness," NASA TM X-3380, 1976.

8. Aydelott, J.C., "Axial Jet Mixing of Ethanol in Cylindrical Containers During Weightlessness," NASA TP-1487, 1979.

9. Aydelott, J.C., "Modeling of Space Vehicle Propellant Mixing," NASA TP-2107, 1983.

10. Sonin, A.A., Shimko, M.A., and Chun, J.H., "Vapor Condensation Onto a Turbulent Liquid. 1. The Steady Condensation Rate as a Function of Liquid-Side Turbulence," International Journal of Heat and Mass Transfer, Vol. 29, No. 9, 1986, pp. 1319-1332.

11. Helmick, M.R., Khoo, B.C., Brown, J.S., and Sonin, A.A., "Vapor Condensation Rate at a Turbulent Liquid Interface, for Application to Cryogenic Hydrogen." AIAA Paper 88-0559. Jan. 1988

12. Jones, W.P., and Launder, B.E.. "The Prediction of Laminarization with a Two-Equation Model of Turbulence," International Journal of Heat and Mass Transfer, Vol. 15, No. 2, Feb. 1972, pp. 301-314.

13. Rodi, W., Turbulence Models and The ir Application in Hydraulics, International Association for Hydraulic Research, The Netherlands, 1980 , pp. 1-92.

14. Launder, B.E., and Spalding, D.B., "The Numerical Computation of Turbulent Flows," Comput. Methods Appl. Mech. Eng., Vol. 3, No. 2. Mar. 1974, pp. 269-289.

15. Chiappetta, L.M., "User's Manual for a TEACH Computer Program for the Analys is of Turbulent, Swirling Reacting Flow in a Research Combustor, R83-915540-27. United Technologies Research Center, East Hartford, CT, Sept. 1983, NASA CR-179547.

16. Escudier, M.P., "The Distribution of MixingLength in Turbulent Flows Near Walls," Report TWF/TN/l. Imperial College of Science and Technology, Dept. of Mechanical Engineering, London, 1966. 
17. Syed, S.A., Chiappetta, L.M., and Gosman, A.D., "Error Reduction Program," PWA-5928-25, Pratt and Whitney Aircraft, East Hartford, CT, Jan. 1985, NASA CR-174775.
18. Issa. R.I.. "Solution of Implicitly Discretised Fluid Flow Equations by Operator-Splitting." Journal of Computational Physics, Vol. 62, No. 1, 1986, pp. 40-65.

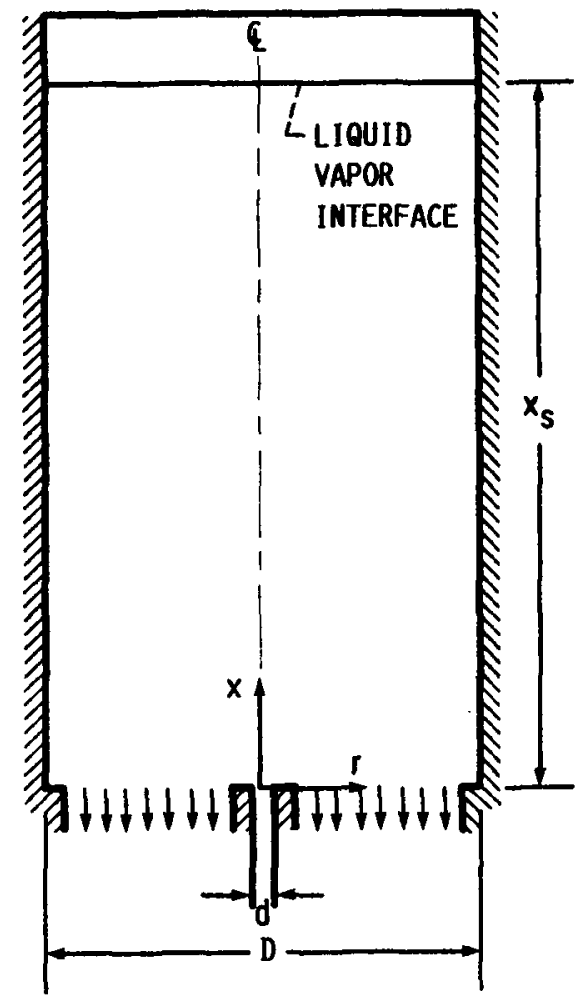

FIGURE 1. - GEOHETRIC CONFIGURATION AND COORDINATES SYSTEM. 


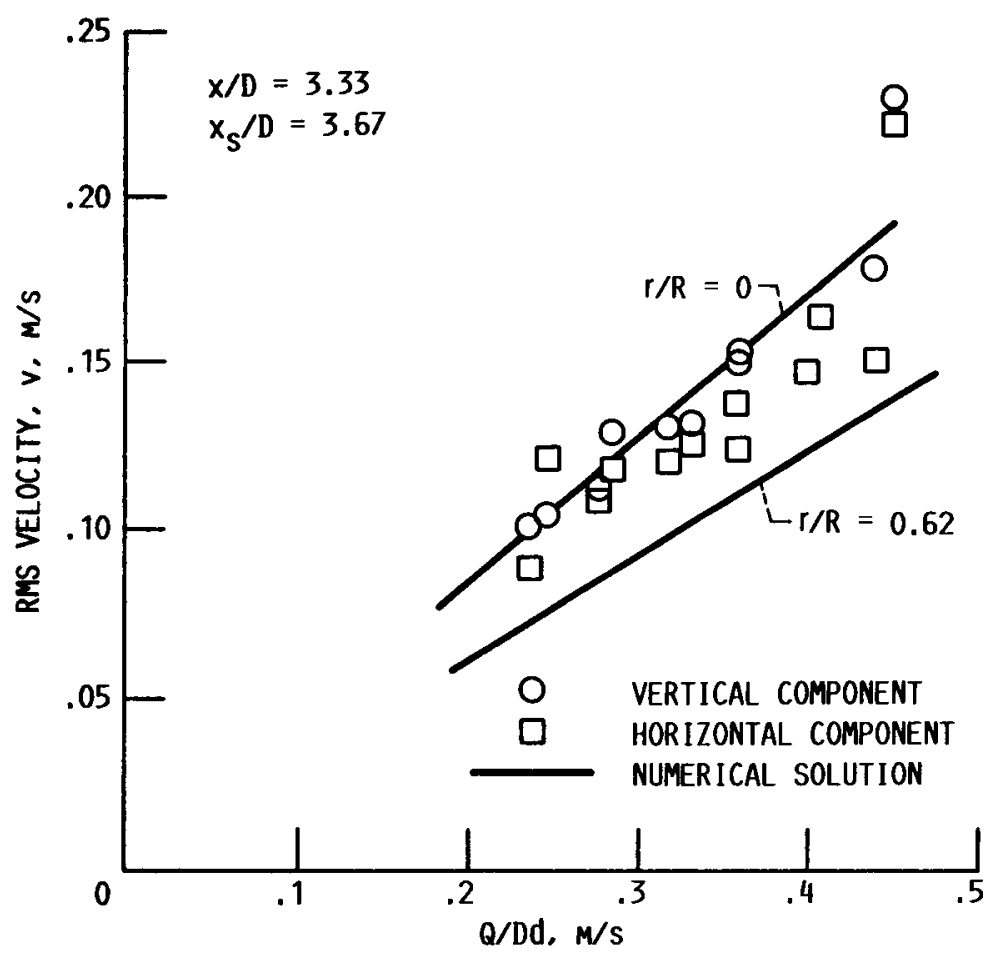

FIGURE 2. - COMPARISON OF CALCULATED $v$ VERSUS Q/Dd WITH THE EXPERIMENT (REF. 10) AT A FIXED AXIAL LOCATION.

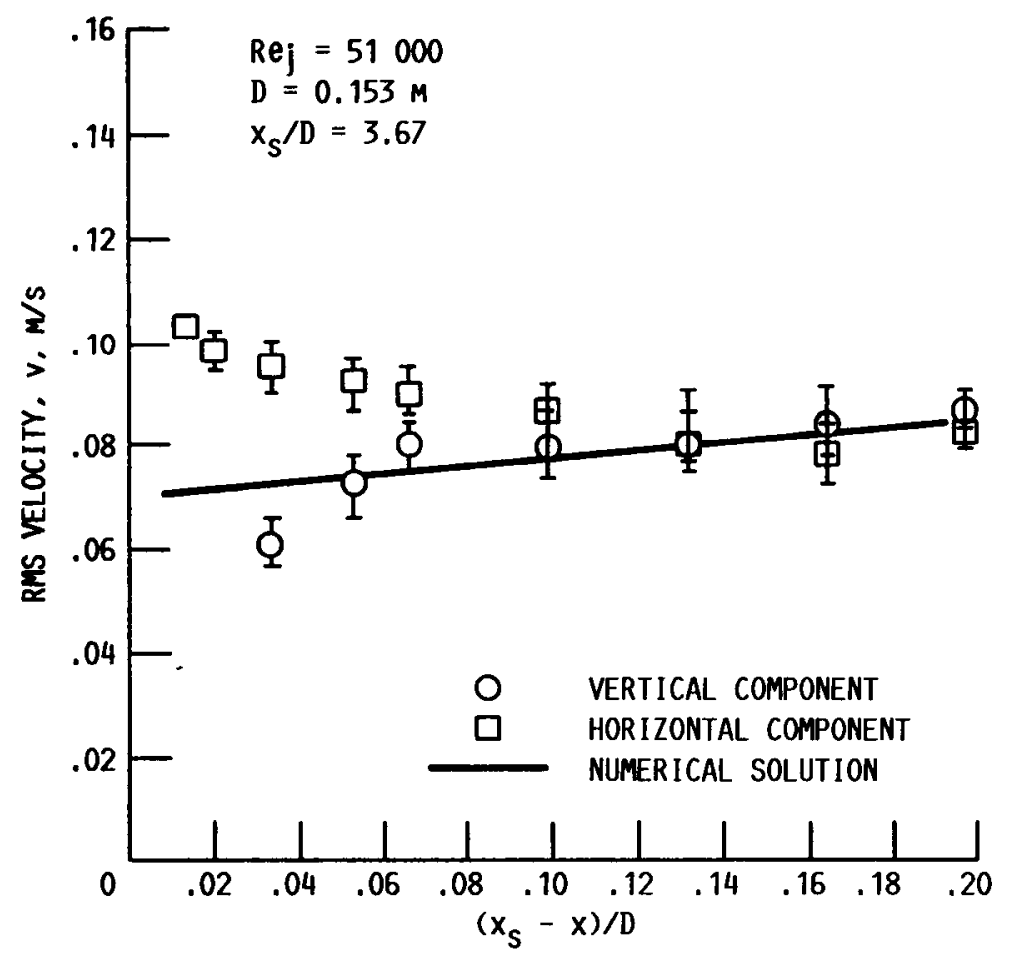

FIGURE 3. - COMPARISON OF CALCULATED $v$ VERSUS DEPTH $\left(x_{s}-x\right)$ BELOW SURFACE WITH EXPERIMENT (REF. 11) AT A FIXED JET FLOW RATE. 


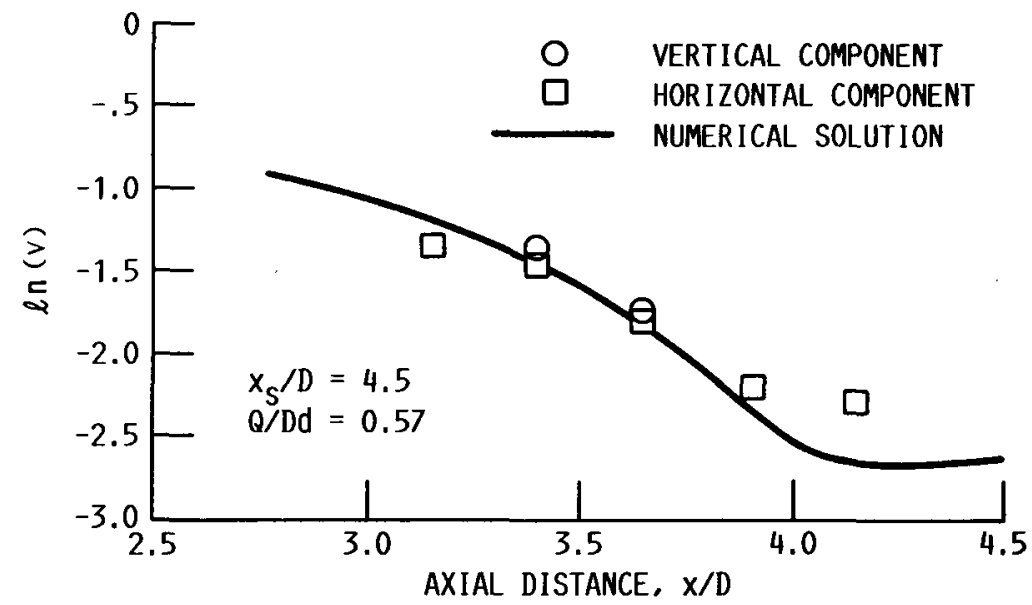

FIGURE 4. - VARIATION OF $v$ WITH THE AXIAL DISTANCE $x / D$. COMPARISON WITH EXPERIMENT (REF. 10).

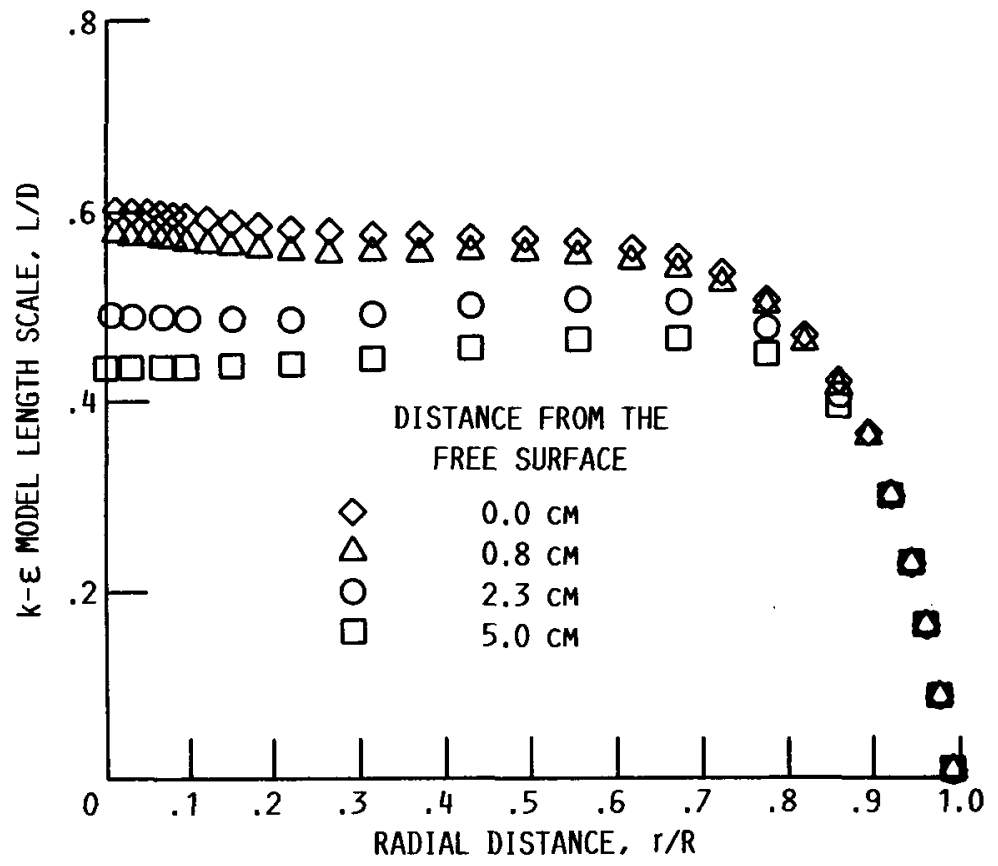

FIGURE 5. - RADIAL DISTRIBUTION OF THE LENGTH SCALE $L=k^{2 / 3} / \varepsilon, \operatorname{Re}_{j}=50000$. 


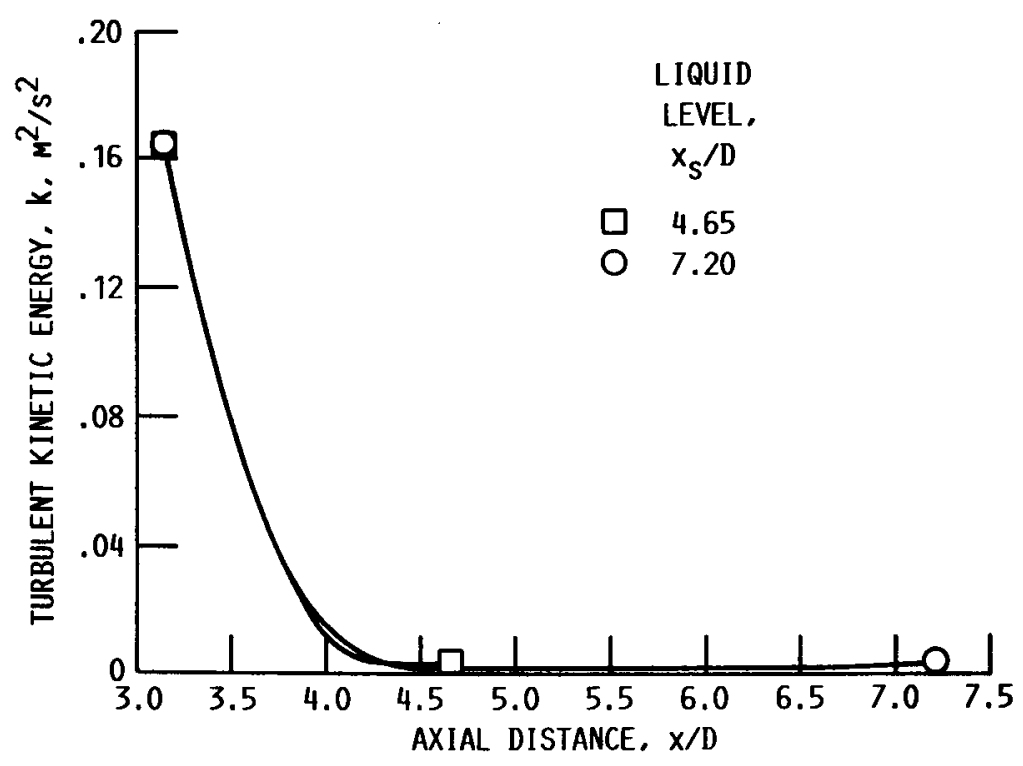

FIGURE 6. - VARIATION OF TURBULENT KINETIC ENERGY WITH THE AXIAL DISTANCE $X / D$.

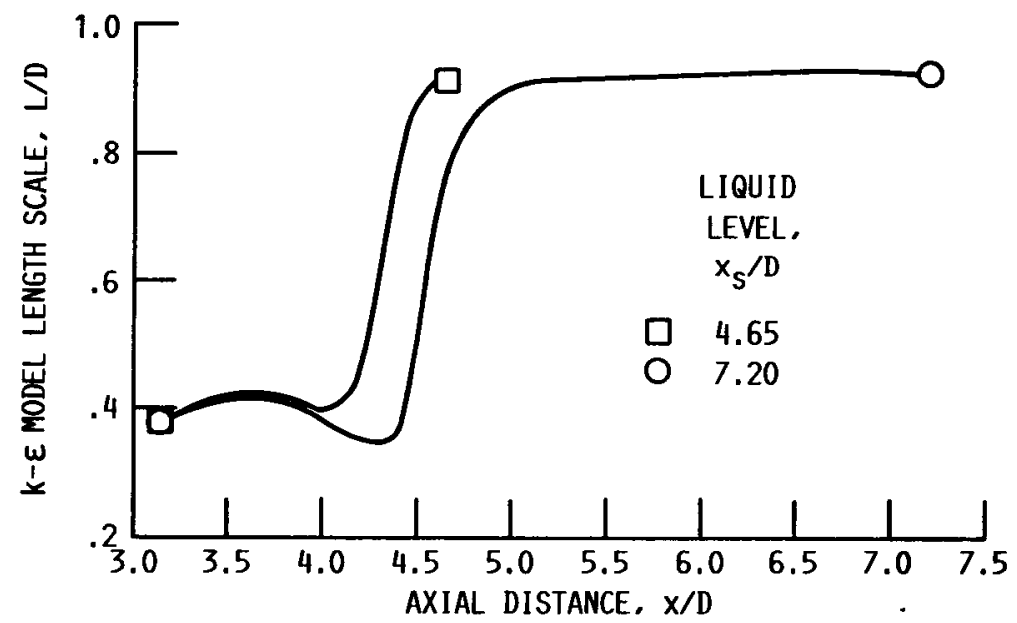

FIGURE 7. - VARIATION OF THE LENGTH SCALE, L, WITH THE AXIAL DISTANCE $X / D$. 


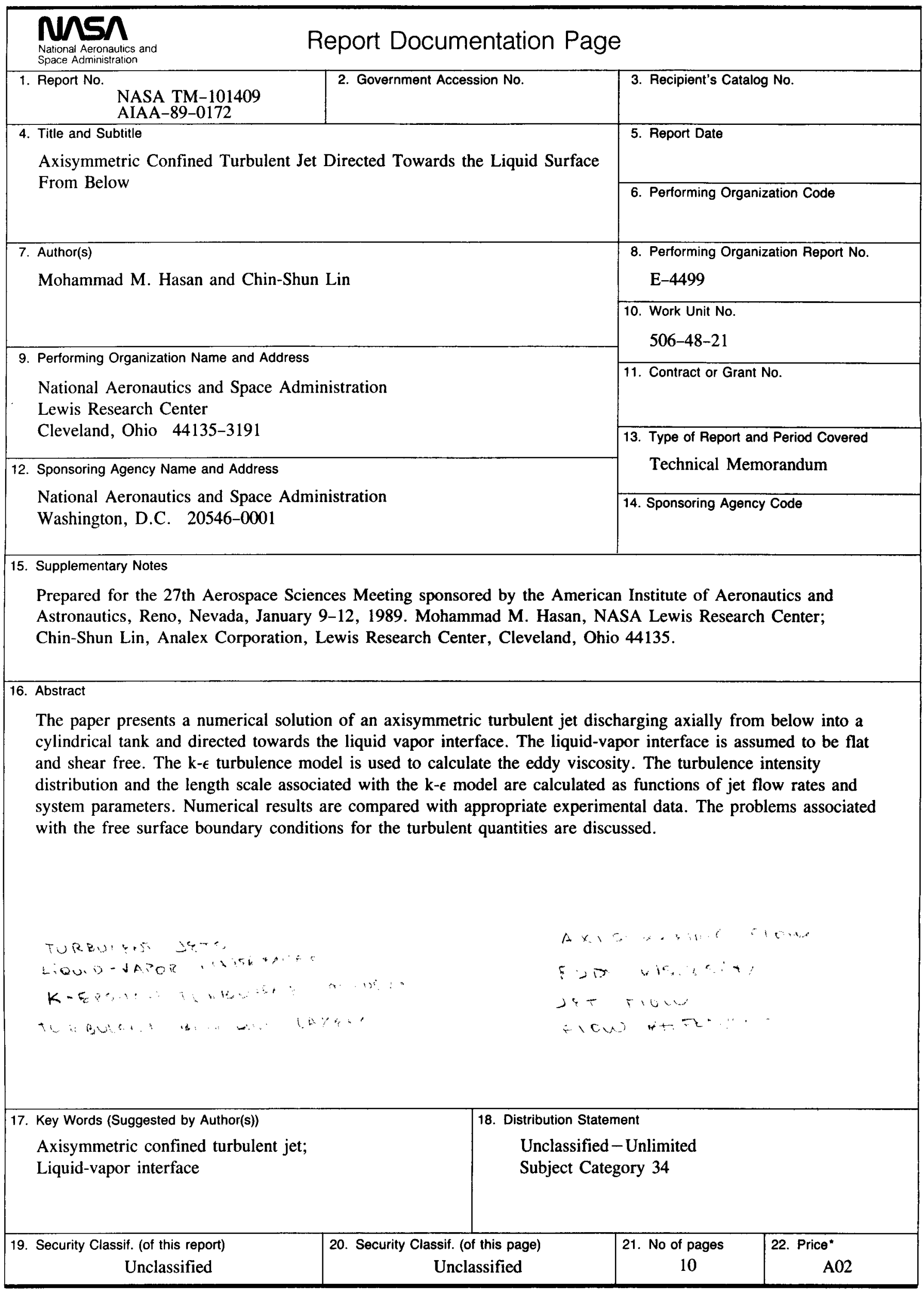

